\title{
Compact design of a redundant manipulator system and application to multiple-goal tasks with temporal constraint
}

\author{
Lounell Bahoy GUETA*, Ryosuke CHIBA**, Tamio ARAI***, Tsuyoshi UEYAMA****, \\ Jose Ildefonso Udang RUBRICO* and Jun OTA* \\ *Research into Artifacts Center for Engineering, The University of Tokyo \\ 5-1-5 Kashiwanoha, Kashiwa, Chiba 277-8568, Japan \\ E-mail: ota@race.u-tokyo.ac.jp \\ ${ }^{* *}$ Research Center for Brain Function and Medical Engineering, Asahikawa Medical University \\ 2-1-1-1 Midorigaoka-higashi, Asahikawa, Hokkaido 078-8510, Japan \\ ***The Center for Promotion of Educational Innovation, Shibaura Institute of Technology \\ 3-7-5 Toyosu, Koto-ku, Tokyo 135-8548, Japan \\ ****DENSO WAVE INCORPORATED, \\ 1 Yoshiike Kusagi Agui-cho, Chita-gun, Aichi 470-2297, Japan
}

Received: 22 November 2016; Revised: 13 February 2017; Accepted: 12 March 2017

\begin{abstract}
This paper aims to design a compact manipulator system consisting of a 6 degree-of-freedom (dof) manipulator and a 1-dof rotary table. It proposes a new compactness measure that considers manipulator swept volume or the space occupied by the system while executing task. Swept volume is crucial because a manipulator has a small footprint but can occupy a substantial space due to its large workspace. Moreover, since compactness can negatively impact manipulator motion, the compact design problem is formulated with motion time or temporal constraint. A best-effort search method is proposed to minimize the swept volume and reduce the motion time to the level of temporal constraint setting. It is carried out by a proposed spatial motion coordination (SMC) for minimizing swept volume and temporal motion coordination (TMC) for reducing motion time. The two schemes are integrated with the base placement optimization, tool attachment optimization and goal rearrangement and are evaluated under various temporal constraint settings. A cross-breed algorithm called STMC utilizing SMC and TMC is then proposed to deal with the limitation. Several optimization frameworks including SMC, TMC and STMC are compared with robot motion simulations. It is found that the ability of SMC and TMC is limited by certain range of the constraint values and STMC is better than the others.
\end{abstract}

Key words : Manipulator, Robot, Engineering optimization, Automation, Operation planning

\section{Introduction}

Space management is a major undertaking in various fields from large-scale infrastructure planning to minute circuitry design. In manufacturing, compact robotic work cell design is essential enabling expansion of operations and easy introduction of latest technologies to adapt to changing demands.

Along with compactness, lead time, the allotted time to manufacture a part on a line (Hopp and Spearman, 2008), has to be satisfied as a temporal constraint. Lead time reduction is a common manufacturing practice (Yagmahan and Yenisey, 2008) to plan product flow and avoid potential bottlenecks. This paper focuses on these two conflicting requirements: work cell compactness and satisfying temporal constraint.

Studies on machine placement in two dimensions have been extensively conducted in facility layout problem (FLP) with constant product flow (Kusiak and Heragu, 1987), (Meller and Gau, 1996), (Singh and Sharma, 2006) and in dynamic FLP (DFLP) with variability in time (McKendall and Hakobyan, 2010). These works focused on inter-work cell layout to minimize material handling cost between work cells. Two issues however require attention in FLP: cintra-work 
cell layout (Wang et al., 2001), which significantly affects the resulting inter-work cell layout, and machine type (Singh and Sharma, 2006), which complicates layout problem. In three-dimensional (3-d) layout problems, applications are found in steel sheet manufacturing (Grangeon et al., 2007), building construction (Jang, H, et al., 2007), component layout (Jang, SH, 2008) and container loading (Yeung and Tang, 2005). In (Cagan et al, 2002), a survey is provided with computational approaches for component rearrangement.

A few works involving manipulators have been conducted in layout problem. In (Shiller, 1997), manipulator motion planning and work cell layout are interactively created by a user. In (Robgoderer and Woenckhaus, 1995), a layout optimization is described involving component arrangement and robot motion simulation. In (Tay and Ngoi, 1996) and (Lueth, 1992), manipulator motion path is estimated as the distance between blocks representing layout area to simplify manipulator motion complexity. Such estimation is limited as manipulator motion time depends on manipulator joint configuration. To minimize manipulator motion time, techniques are proposed like motion planning, manipulator base placement optimization (Hsu et al., 1999), (Gueta et al., 2007), goal rearrangement (Saha et al., 2006), and task-based manipulator optimization (Kim and Khosla, 1993). Similar to 2-d FLP, the base placement optimization designs the manipulator base position relative to goals. The task-based manipulator optimization proposes manipulator design based on a given task. In (Gueta et al., 2008), a method is proposed by adding a tool attachment, a linkage at the manipulator flange, as a more cost-effective solution compared to task-based manipulator optimization.

The above previous studies have focused individually on either designing a compact manipulator system as a spatial requirement or minimizing manipulator motion time as a temporal requirement. This paper considers jointly these two requirements.

As the objective, a work cell involving a manipulator system has to be designed compact with a temporal constraint. The work cell consisting of a 6-degree-of-freedom (dof) manipulator with a tool and a 1-axis rotary table is utilized as an example. When the manipulator reaches a goal, it sweeps a volume in space. Similarly, as the table rotates, the work piece along with the table temporally occupies a region in space. This total occupied space by the manipulator system and work piece at home position (when no task is being done) and during task execution is referred to as swept volume. We introduce the motion time as the temporal constraint since it is significant in real manufacturing to achieve a userinput lead time, $t_{\text {desired }}$. More significantly, we formulate a compactness measure based on the swept volume because the manipulator system has a small footprint but can occupy a substantial space due to its large workspace.

The three key challenges in this study are stated as:

(a) Designing an effective solution to achieve minimal 3-d swept volume.

(b) Finding a good-quality solution from a prohibitively large, complex search space.

(c) Satisfying a constraint conflicting with the desired objective function.

Challenge (a) deals with compactness in 3-d layout problem along with complicated manipulator motion. Previous 3-d layout problems extend typical 2-d FLP by considering horizontal and vertical material movements. This study deals with a more complicated movement described by position and orientation (pose) change of connected manipulator linkages. Challenge (b) stems from the manipulator kinematic redundancy wherein the manipulator can have multiple candidate configurations with equal swept volume. This is similar to selecting a node in a graph problem with equallyweighted edges. Challenge (c) deals with the problem of simultaneously satisfying the temporal constraint and designing a compact system. For example, in manipulator base placement optimization, a manipulator must generally be placed far from its goals to achieve short motion time (Gueta et al., 2007), inadvertently resulting to a large covered floor area. Furthermore, satisfying the constraint has several scenarios with two extreme cases: an unconstrained optimization and an optimization too restricted by the constraint. These three challenges are respectively dealt with as follows:

i. A spatial motion coordination (SMC) to minimize swept volume

ii. A decision logic embedded in a search methodology for manipulator configuration selection.

iii. A cross-breed of two motion coordination schemes (STMC) to satisfy the temporal constraint while designing a compact system.

The SMC is a new scheme for motion planning, earlier presented in (Gueta et al., 2009); whereas, existing motion coordination scheme, referred to as temporal motion coordination (TMC), considers only motion time (Jouaneh et al., 1990), (Gueta et al., 2011). Moreover, previous techniques such as base placement optimization, goal rearrangement, tool 
attachment optimization are evaluated and integrated with SMC in this study. Although these previous techniques are capable of minimizing the swept volume, simulation results showed that SMC, as the core solution, significantly minimizes it.

The search methodology is a best-effort method to select manipulator configuration that minimizes swept volume and reduce the motion time, whenever possible. The selection is based on minimizing the swept volume and reducing the motion time to $t_{\text {desired }}$ level, when multiple equally-weighted configurations are available.

In simulation results, it is shown that SMC and TMC are capable of only satisfying certain ranges of $t_{\text {desired }}$ values. Hence, STMC is proposed as a cross-breed of SMC and TMC. STMC is a practical solution to be able to minimize swept volume and satisfy $t_{\text {desired }}$ constraint which are shown to be conflicting requirements in the simulation.

Section 2 formulates the compact design problem. Section 3 describes the optimization framework. Section 4 discusses the search methodology for motion coordination. Section 5 provides the simulations and results. Section 6 concludes this paper.

\section{Problem formulation and analysis}

\subsection{Problem formulation}

The problem is formulated in the context of point-point multiple-goal task realization seen in inspection and assembly tasks. A manipulator system is tasked to reach a given number of goals. See Figure 1 where $\mathrm{L}_{0}, \ldots, \mathrm{L}_{7}$ are the reference frames of the manipulator system. $\mathrm{L}_{0}$ denotes the reference frame of the rotary table base, $\mathrm{L}_{1}$ to that of the manipulator base, $\mathrm{L}_{6}$ to that of the manipulator flange where a tool attachment is connected and $\mathrm{L} 7$ to that of the tool tip. This system is utilized due to its inherent advantages such as flexibility, efficient use of workspace and short motion time (Jouaneh et al., 1990). The table is crucial because the manipulator dimensions are comparable to that of the work piece. With 1-dof of redundancy, controlling the system is simpler compared to that with higher degree of freedoms (dofs).

The input parameters are assumed to be as follows:

- Manipulator system $A$ with known Denavit-Hartenberg (D-H) parameters shown in Table 1 and 3-d model

- Work piece $B$ with known size and shape which is placed on the rotary table.

- Goal position and orientation $g_{i}, i=(1, \ldots, \mathrm{n})$ where $\mathrm{n}$ is the number of goals.

- Initial (final) position and orientation of $A$ before (after) the task execution.

In this study, the initial and final manipulator pose (i.e., $g_{0}$ and $g_{n+1}$ ) are the same. The configuration $q_{i}=$ $\left(\theta_{i}^{0}, \ldots, \theta_{i}^{6}\right), i=(0, \ldots, \mathrm{n})$ denotes the manipulator configuration at $g_{i}$, where $\theta_{i}^{0}$ is the table angle and $\theta_{i}^{1}, \ldots, \theta_{i}^{6}$ are the manipulator joint angles. The motion time of $A$ from $g_{0}$ to $g_{i}$ is expressed by:

$$
t_{i}=\sum_{k=1}^{i} c\left(g_{k-1}, g_{k}\right) \text {. }
$$

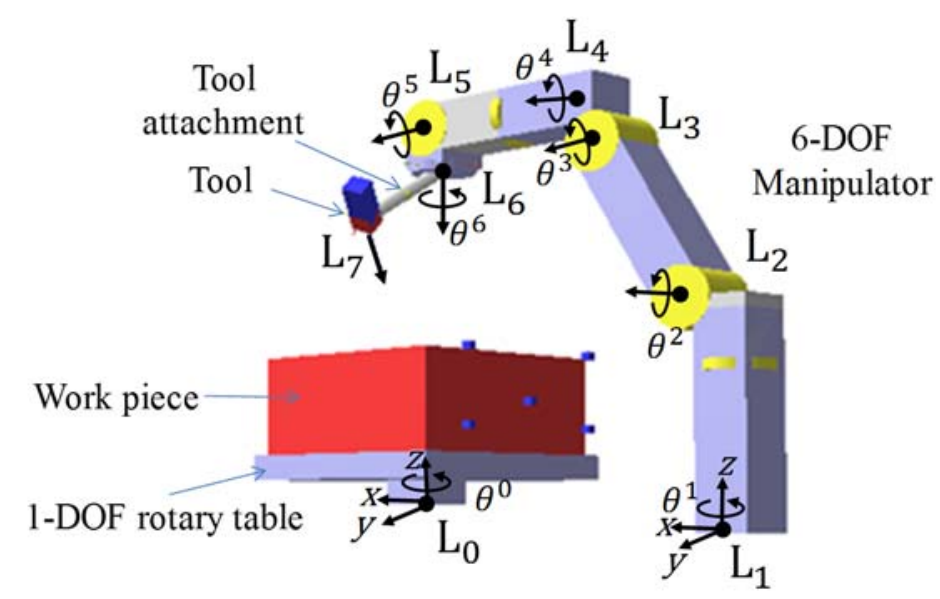

Fig. 1 The redundant manipulator system. 
Gueta, Chiba, Arai, Ueyama, Rubrico and Ota,

Journal of Advanced Mechanical Design, Systems, and Manufacturing, Vol.11, No.2 (2017)

Table $1 \mathrm{D}-\mathrm{H}$ parameters of the manipulator

\begin{tabular}{|c|c|c|c|c|}
\hline Joint number $j$ & $d_{j}[\mathrm{~mm}]$ & $a_{j}[\mathrm{~mm}]$ & $\alpha_{j}[\mathrm{rad}]$ & $\theta^{j}$ \\
\hline 1 & 335 & 75 & $-\frac{\pi}{2}$ & $\theta^{1}$ \\
\hline 2 & 0 & 365 & 0 & $\theta^{2}$ \\
\hline 3 & 0 & 90 & $-\frac{\pi}{2}$ & $\theta^{3}$ \\
\hline 4 & 405 & 0 & $\frac{\pi}{2}$ & $\theta^{4}$ \\
\hline 5 & 0 & 0 & $-\frac{\pi}{2}$ & $\theta^{5}$ \\
\hline 6 & 80 & 0 & 0 & $\theta^{6}$ \\
\hline
\end{tabular}

Where $\mathrm{c}(\cdot)$ is the motion time between two goals. The total motion time from $g_{0}$ to $g_{\mathrm{n}+1}$ is denoted as $t_{\mathrm{n}+1}$. The swept volume, the volume swept by $A$ during movement and that of $B$ when rotating, is defined as:

$$
S_{i}^{\alpha}=\bigcup_{t=0}^{t_{i}} s^{\alpha}(t) .
$$

Where $\alpha=(\mathrm{A}, \mathrm{B}), s^{\alpha}(t)$ is the occupied volume at time $t$ and $S_{i}^{\alpha}$ is the union of the swept volumes from $t=0$ to $t_{i}$. The total swept volume of $A$ and $B$ during task execution can be expressed as $S_{\mathrm{n}+1}^{A}$ and $S_{\mathrm{n}+1}^{B}$, respectively.

The performance index $f$ is calculated as:

$$
f=\min \left(S_{\mathrm{n}+1}^{A} \cup S_{\mathrm{n}+1}^{B}\right)
$$

Subject to the following constraints:

- Collision constraint

$$
s^{A}(t) \cap s^{B}(t)=0, t=\left[0, \ldots, t_{\mathrm{n}+1}\right] .
$$

Equation 4 is utilized so that any potential collision between $A$ and $B$ is prevented.

- Temporal constraint

$$
t_{\mathrm{n}+1} \leq t_{\text {desired }}
$$

- $\quad$ Other problem-specific constraints

Constraints such as joint and velocity limits are imposed. Moreover, the calculation time, CalcTime, is limited by maximum time, maxCalcTime, for practical reasons.

\section{CalcTime $\leq$ maxCalcTime}

The design parameters are:

- base position $X_{L_{1}}^{L_{0}}$,

- tool attachment $X_{L_{7}}^{L_{6}}$

- goal order $\pi$

- configuration $q_{i}, i=(0, \ldots, \mathrm{n})$.

where $X_{L_{1}}^{L_{0}}=\left(x_{L_{1}}^{L_{0}}, y_{L_{1}}^{L_{0}}, z_{L_{1}}^{L_{0}}\right)$ denotes the parameters defining the manipulator base position relative to the table position, $X_{L_{7}}^{L_{6}}=\left(x_{L_{7}}^{L_{6}}, y_{L_{7}}^{L_{6}}, z_{L_{7}}^{L_{6}}, \alpha_{L_{7}}^{L_{6}}, \beta_{L_{7}}^{L_{6}}, \gamma_{L_{7}}^{L_{6}}\right)$ define the transformation matrix between manipulator flange and tool tip where $x_{L_{7}}^{L_{6}}, y_{L_{7}}^{L_{6}}, z_{L_{7}}^{L_{6}}$ are the $x, y$, and $z$ coordinates while $\alpha_{L_{7}}^{L_{6}}, \beta_{L_{7}}^{L_{6}}, \gamma_{L_{7}}^{L_{6}}$ are the roll, pitch and yaw, respectively. The parameter $\pi$ is a permutation of $g_{i}, i=(1, \ldots, \mathrm{n})$ and $q_{i}$ is the manipulator system configuration at goal $i$.

\subsection{Problem analysis}

The possible design solutions are numerous and have to be derived from sub-problems with unique characteristics. The base placement and tool attachment optimizations are nonlinear complex problems due to manipulator kinematics 
and collision occurrence. The mathematical relation of design parameters and swept volume cannot be expressed as a closed-form function; the derivative is too complicated to be calculated. A suitable solution is then a derivative-free direct search method. To reduce the solution search space dimension, the base placement and tool attachment parameters can be made discrete values. Suppose the number of parameter (i.e., the $\boldsymbol{x}-, \boldsymbol{y}-$ or $\boldsymbol{z}-$ parameter of the base placement) is $\boldsymbol{d}$. The total number of possible solutions is $\boldsymbol{d}^{3}$. Similarly, in the tool attachment optimization, the total number of solutions can be denoted as $\boldsymbol{e}^{\mathbf{6}}$ where $\boldsymbol{e}$ is the number of one parameter. On the other hand, the goal rearrangement is a combinatorial problem with $\mathbf{n}$ ! solutions. To resolve the kinematic redundancy (i.e., the inverse kinematics solutions is infinite), motion coordination between the manipulator and table can be utilized. The potential collision between A and $B$ in 3-d environment complicates the manipulator configuration selection. To reduce the problem complexity, the manipulator configuration can be parameterized into one variable, which is the table rotational angle (See Section 4 for details). Without a mathematical expression to define potential collision, $\boldsymbol{\theta}_{\boldsymbol{i}}^{\mathbf{0}}$ values are made discrete values and a graph search method can be performed to derive collision-free path. If $\boldsymbol{\theta}_{\boldsymbol{i}}^{\mathbf{0}}$ has a resolution of $\mathbf{r}$, then the total configurations for $\mathbf{n}$ goals is $\mathbf{r}^{\mathbf{n}}$. Overall, the total number of solutions is $\boldsymbol{d}^{\mathbf{3}} \boldsymbol{e}^{\mathbf{6}} \mathbf{n} ! \mathbf{r}^{\mathbf{n}}$. For values of $\boldsymbol{d}=100, \boldsymbol{e}=100, \mathbf{n}=30$ and $\mathbf{r}=64$, it is equivalent to $2.79 \times \mathbf{1 0}^{\mathbf{1 0 3}}$ showing the high dimensionality of the problem.

\section{Optimization framework}

In (Gueta et al., 2011), we proposed a framework integrating the base placement optimization, goal rearrangement and motion coordination. It was utilized to minimize manipulator motion time. That framework is applicable in this study for the following reasons. (a) The manipulator base placement dictates the effective floor area occupied by the manipulator system (b) The goal order affects the manipulator swept volume. (c) The configurations from the motion coordination can be selected to minimize the swept volume. This study extends the framework by integrating the tool attachment optimization.

Figure 2 shows the framework consisting of two stages: (a) the base placement and tool attachment optimizations and (b) the goal rearrangement and motion planning. First, the manipulator base placement relative to the table is optimized and a tool attachment is designed. Then, a goal order is derived in the goal rearrangement while the manipulator system motion is coordinated. The motion time $t_{\mathrm{n}+1}$ is calculated and compared to $t_{\text {desired }}$, which, being a constraint, is imposed before the work cell size is calculated. Iterations are performed until maxCalcTime is reached. The base placement and tool attachment optimizations both involve parameter optimizations of their corresponding transformation matrices. These optimizations precede the motion coordination to define the manipulator system kinematic equation necessary in calculating manipulator configurations. The goal rearrangement is solved simultaneously with the motion coordination because the manipulator configurations are highly dependent on a goal order. Since a calculation time limit is imposed, exploring all possible solutions is not possible. Hence, algorithms have to be selected on the basis of deriving good-quality solution within a reasonable amount of calculation time.

The remaining parts of this section briefly describe the base placement optimization, tool attachment optimization (Subsection 3.1), and goal rearrangement (Subsection 3.2). For detailed discussion, refer to our previous work (Gueta et al., 2011). The motion time calculation and compactness evaluation are discussed in Subsection 3.3 and Subsection 3.4, respectively.

\subsection{Base placement and tool attachment optimizations}

One consideration in the base placement optimization is the manipulator massive structure; placing it on a wall or a ceiling limits its possibility for relocation. These scenarios are avoided by constraining the manipulator and table to be vertically positioned and designing only the manipulator base translation parameters. In the tool attachment optimization, a tool attachment is designed to be a fixed linkage without moving parts. It can be designed with extra dofs, which complicates manipulator motion planning. Simulated annealing algorithm (SA) is employed due to its robustness to local minima (Kirkpattrick et al., 1983). In this study, it is too difficult to determine whether a solution is optimal or not; therefore, SA parameters are selected to derive a good quality solution within the calculation time limit. For practical setting of SA parameters, refer to (Park and Kim,1998).

\subsection{Goal rearrangement with clustering method}


In the goal rearrangement, goals are clustered based on their locations on $\boldsymbol{B}$. The maximum cluster number is 5 assuming that $\boldsymbol{B}$ can be approximately represented as a rectangular box. The clusters are rearranged using 2-opt algorithm and the Lin-Kernighan (LK) heuristic is used to derive goal order within each cluster (Helsgaun, 2000). The goal rearrangement is terminated when a solution is not improved or when maxCalcTime is exceeded. Goals are clustered to reduce the possibly long calculation time since the number of goal orders increases exponentially with $\mathbf{n}$.

\subsection{Motion time calculation}

In calculating $\boldsymbol{t}_{\boldsymbol{i}}$, the maximum joint velocity profile is utilized for simplicity where:

$$
\mathbf{c}\left(g_{k-1}, g_{k}\right)=\max _{j=0, \ldots, 6}\left(\frac{\left|\theta_{k}^{j}-\theta_{k-1}^{j}\right|}{v^{j, m a x}}\right)
$$

where $\boldsymbol{\theta}_{\boldsymbol{k}}^{\boldsymbol{j}}$ is the joint $\boldsymbol{j}$ angle at goal $\boldsymbol{g}_{\boldsymbol{k}}$ and $\boldsymbol{v}^{\boldsymbol{j}, \boldsymbol{m a x}}$ is the maximum speed of joint $\boldsymbol{j}$. To consider manipulator joint acceleration, a trapezoidal velocity profile can be employed; refer to our work in -(Gueta et al., 2007). Note that $\boldsymbol{t}_{\boldsymbol{n}+\mathbf{1}}$ is calculated after $\boldsymbol{\theta}_{\boldsymbol{i}}^{\mathbf{0}}$ values are derived as a result of point-to-point motion. Therefore, checking whether $\boldsymbol{t}_{\boldsymbol{d e s i r e d}}$ constraint is satisfied or not is conducted after calculating $\boldsymbol{t}_{\boldsymbol{n}+\mathbf{1}}$.

\subsection{Compactness evaluation}

Calculating the exact shape of the swept volume can be computationally expensive. Furthermore, a very detailed shape of the swept volume is impractical since work cells are typically designed to be rectangular for modularity reasons. This study proposes a practical method by finding the least-sized box bounding the swept volumes of $\boldsymbol{A}$ and $\boldsymbol{B}$. The swept volume is then derived by finding the box dimensions in the Cartesian space.

A calculation result example is shown in Figure 3. Twenty goals shown by blue dots exist with 4 goals on each face of $\boldsymbol{B}$. A column shows the manipulator configuration (top) and the swept volume (bottom). Fig. 3(a) bottom is the swept volume approximated by the bounding box enclosing $\boldsymbol{A}$ and $\boldsymbol{B}$. The blue outlines indicate the approximated swept volumes dimensions: the darker ones are the currently calculated dimensions while the lighter ones are the dimensions from the preceding manipulator position.

\section{Search methodology for motion coordination 4.1 Overview of TMC and SMC}

Previous motion coordination schemes only consider temporal requirement (Jouaneh et al., 1990),(Gueta et al.,

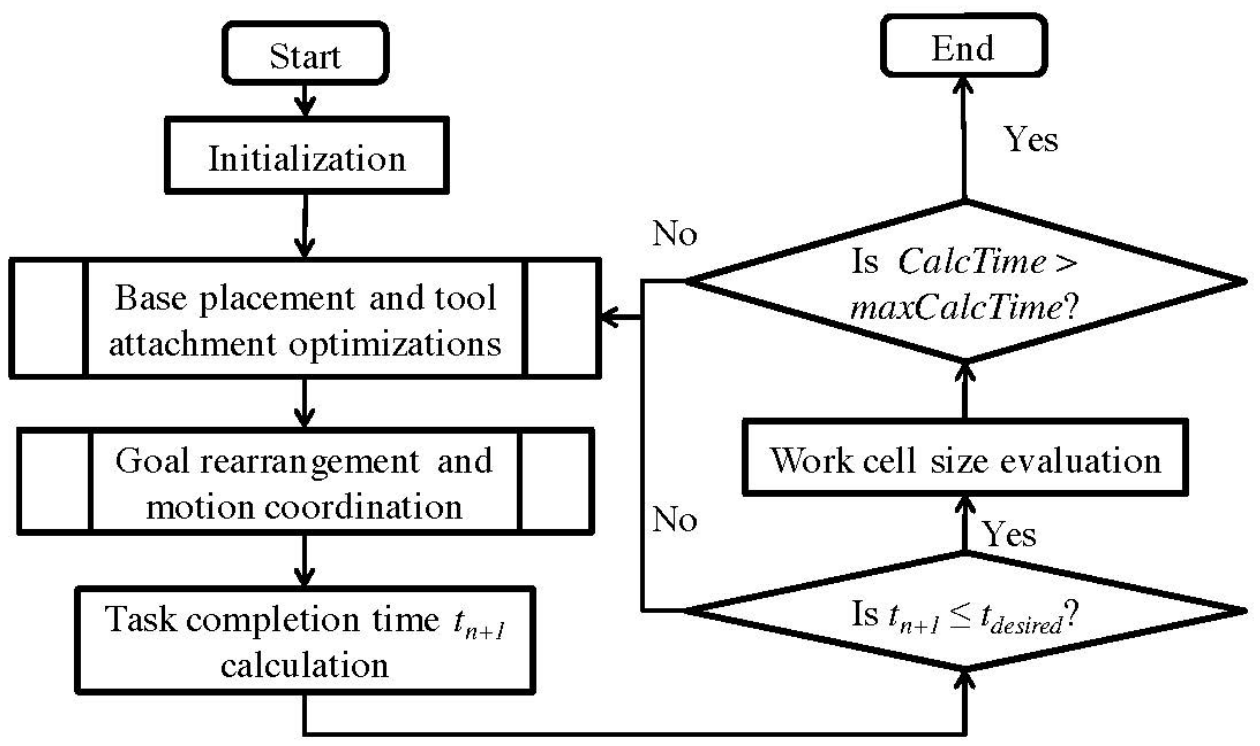

Fig.2 Optimization framework. 
2011). In this study, the motion coordination is more complicated since it considers jointly spatial and temporal requirements. The manipulator and table are synchronously coordinated to deal with manipulator system kinematic redundancy and potential collision.

Two motion coordination schemes are implemented and evaluated: the temporal motion coordination (TMC) and spatial motion coordination (SMC). In TMC, configurations are selected to minimize motion time by searching the configuration space and selecting the configuration with the least $\mathbf{c}\left(\boldsymbol{g}_{\boldsymbol{i}-\mathbf{1}}, \boldsymbol{g}_{\boldsymbol{i}}\right)$. While in SMC, configurations are selected to minimize swept volume by evaluating the Cartesian space and selecting the configuration with the least swept volume. The solution search space of manipulator configuration selection is represented as a weighted graph. In planning manipulator motion from $\boldsymbol{g}_{\mathbf{0}}$ to $\boldsymbol{g}_{\mathbf{n}}$, the table can be rotated at various $\boldsymbol{\theta}_{\mathbf{0}}^{\boldsymbol{i}}$. Figure 4(a) and Figure 4(b) illustrate the search space representation with $\mathbf{n}+\mathbf{2}$ stages with the first and last stages corresponding to $\boldsymbol{g}_{\mathbf{0}}$. A node represents a goal rotated at a certain table angle. A row represents a particular goal at several table angles calculated based on (8) and (9):

$$
\begin{gathered}
\theta_{0}^{i}(l)=\theta^{0, \min }+d(\mathrm{r}) l \mathrm{r}, l=\left(0, \frac{1}{\mathrm{r}}, \frac{2}{\mathrm{r}}, \ldots, 1\right) \\
d(\mathrm{r})=\frac{\theta^{0, \max }-\theta^{0, \min }}{\mathrm{r}}, \mathrm{r} \geq 1
\end{gathered}
$$

where $\boldsymbol{\theta}_{\mathbf{0}}^{\boldsymbol{i}}(\boldsymbol{l})$ is the table angle, $\mathbf{r}$ is a user-defined resolution of the table rotation step, $\mathbf{d}$ is the step, $\boldsymbol{\theta}^{\mathbf{0}, \boldsymbol{m i n}}$, and $\boldsymbol{\theta}^{\mathbf{0}, \boldsymbol{m a x}}$ are the minimum and maximum search limit values of $\boldsymbol{\theta}_{\boldsymbol{i}}^{\mathbf{0}}$.

The graph edge is weighted depending on the scheme. In SMC, the weight is defined by:

$$
\Delta \mathbf{S}\left(\boldsymbol{g}_{i-1}, \boldsymbol{g}_{i}\right)=\left(\boldsymbol{S}_{i}^{A} \cup \boldsymbol{S}_{i}^{B}\right)-\left(\boldsymbol{S}_{i-1}^{A} \cup \boldsymbol{S}_{i-1}^{B}\right)
$$

where $\Delta \mathbf{S}\left(\boldsymbol{g}_{\boldsymbol{i}-\mathbf{1}}, \boldsymbol{g}_{\boldsymbol{i}}\right)$ is the increase in the swept volume from $\boldsymbol{g}_{\boldsymbol{i}-\mathbf{1}}$ to $\boldsymbol{g}_{\boldsymbol{i}}$.

In TMC, the weight is the point-to-point motion time $\mathbf{c}\left(\boldsymbol{g}_{\boldsymbol{i}-\mathbf{1}}, \boldsymbol{g}_{\boldsymbol{i}}\right)$ using Eq. (7). Figure 4(a) represents the graph for SMC (spatial graph) while Fig. 4(b) is that for TMC (temporal graph). For the search space complexity, we provide similar analysis in (Gueta et al., 2011).

\subsection{Decision logic for equally-weighted manipulator configurations}

A decision logic is important for the purpose of minimizing swept volume while satisfying a temporal constraint. It is a best-effort method since it tries to select a configuration with the least swept volume and minimum motion time, although having no guarantee that it would satisfy the temporal constraint. Suppose in Fig. 4(a) the manipulator from $\boldsymbol{g}_{\mathbf{1}}$ to $\boldsymbol{g}_{\mathbf{2}}$ have several candidate configurations with equal swept volumes, represented by edges emanating from $\boldsymbol{g}_{\mathbf{1}}$. The design logic enables selection of one distinct configuration. As shown in Fig. 4(c), SMC searches solutions at the spatial graph. If $\boldsymbol{g}_{\boldsymbol{i}}$ has several configurations with equal $\Delta \mathbf{S}\left(\boldsymbol{g}_{\boldsymbol{i}-1}, \boldsymbol{g}_{\boldsymbol{i}}\right)$, SMC searches at the temporal graph to select the configuration with minimum $\mathbf{c}\left(\boldsymbol{g}_{\boldsymbol{i}-\mathbf{1}}, \boldsymbol{g}_{\boldsymbol{i}}\right)$ from these possible configurations.

In the TMC case, the optimization framework still minimizes the swept volume except that in the motion coordination, TMC is used to reduce the calculation time. If TMC evaluates that the minimum $\mathbf{c}\left(\boldsymbol{g}_{\boldsymbol{i}-\mathbf{1}}, \boldsymbol{g}_{\boldsymbol{i}}\right)$ corresponds to more than one configuration, it will search the spatial graph to select one with the least $\Delta \mathbf{S}\left(\boldsymbol{g}_{\boldsymbol{i}-\mathbf{1}}, \boldsymbol{g}_{\boldsymbol{i}}\right)$.

Note that the decision logic is only applied between consecutive goals since it is appropriate to the nearest neighbor algorithm (NNA) algorithm we employed for graph traversal. This logic can be extended to other algorithms such as Dijkstra algorithm (DA). The NNA selects the immediate edge with the least weight value; see (Gueta et al., 2011) for the algorithm details. It is utilized due to its low computational requirement. We showed that NNA, DA, and the combination of NNA and DA have corresponding tradeoffs in the calculation time and solution quality. The DA obtains high quality solution but with long calculation time. The NNA is the fastest with good-quality solution.

\subsection{Comparison between TMC and SMC}

The motivation for utilizing SMC and TMC is anchored on three scenarios with respect to $\boldsymbol{t}_{\text {desired }}$ settings: (i)

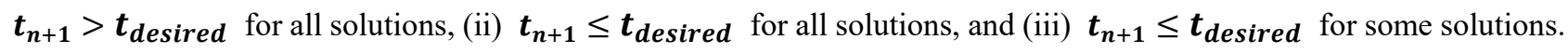


Scenarios (i) and (ii) are extreme cases wherein $\boldsymbol{t}_{\text {desired }}$ value is not realistic in (i) while optimization is effectively unconstrained relative to $\boldsymbol{t}_{\text {desired }}$ in (ii). In (iii), $\boldsymbol{t}_{\boldsymbol{n}+\boldsymbol{1}}$ can be initially larger than $\boldsymbol{t}_{\boldsymbol{d e s i r e d}}$ and the inequality is eventually satisfied after optimization is performed. These three cases however cannot be distinguished without performing optimization. To observe example cases, a simulation is conducted performing only motion coordination to examine SMC and TMC; $\mathbf{n}=\mathbf{2 0}$ goals with 4 goals on each face of the work piece. Figure 5 shows the simulation result. Comparing the motion time and swept volume, SMC has larger $\boldsymbol{t}_{\boldsymbol{n}+\mathbf{1}}$ than TMC but with smaller $\boldsymbol{f}$ value. Similar results are observed for other $\mathrm{n}$ values. From these results, we hypothesized that in designing a compact system with temporal constraint it is possible that for specific temporal constraints TMC can be more appropriate than SMC and vice versa. If $\boldsymbol{t}_{\text {desired }}$ value is large (s.t. $\boldsymbol{t}_{\boldsymbol{n}+\mathbf{1}} \leq \boldsymbol{t}_{\text {desired }}$ for all solutions), or the optimization has no $\boldsymbol{t}_{\text {desired }}$ constraint, SMC is more appropriate than TMC.
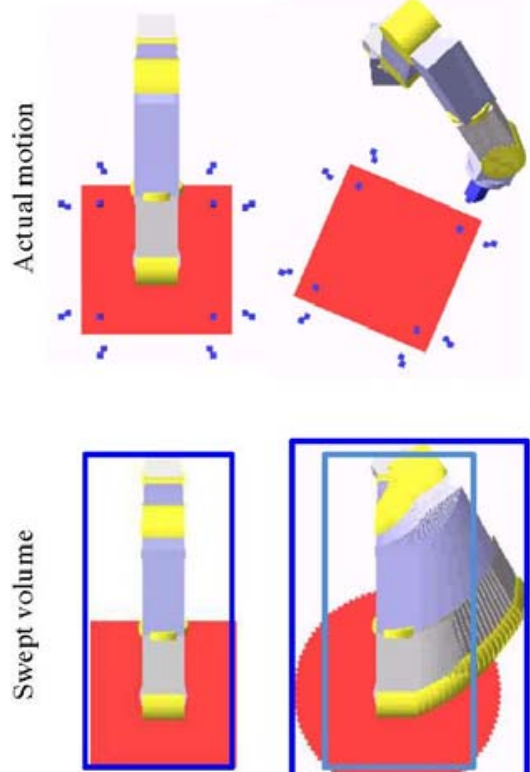

(a)

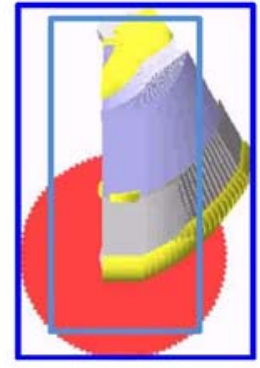

(b)
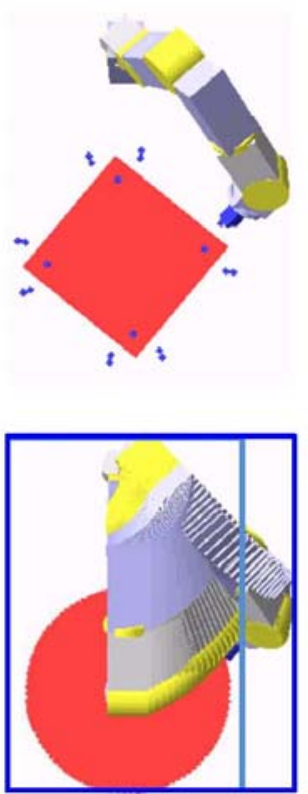

(c)
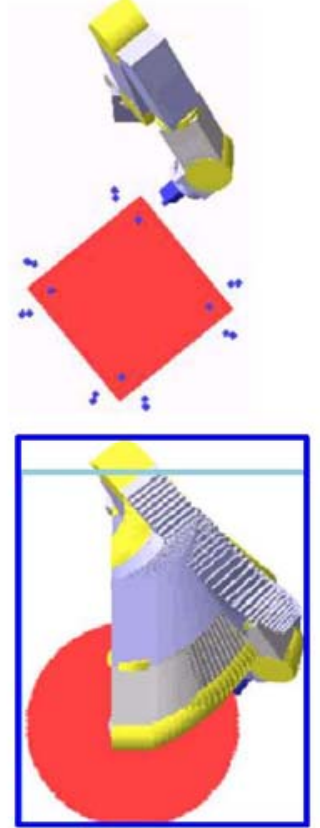

(d)
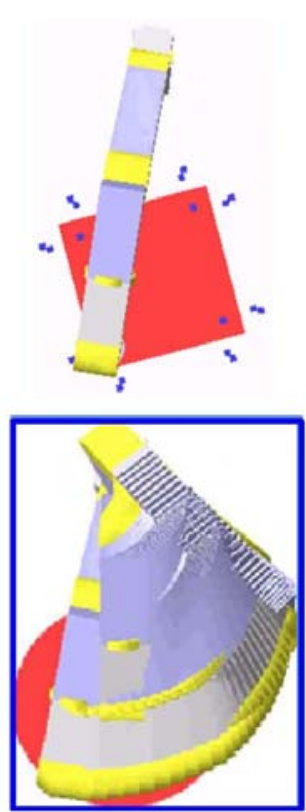

(e)

Fig. 3 Swept volumes (Top view).

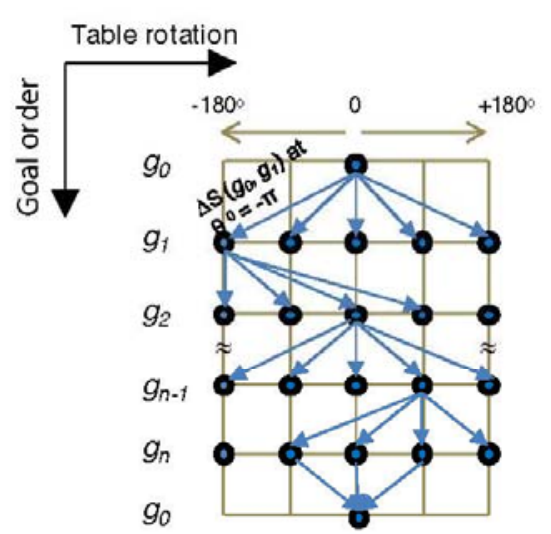

(a) Spatial graph

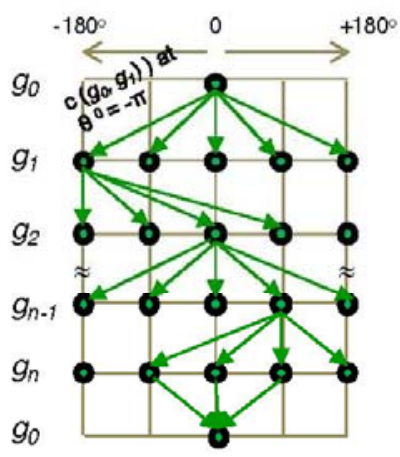

(b) Temporal graph

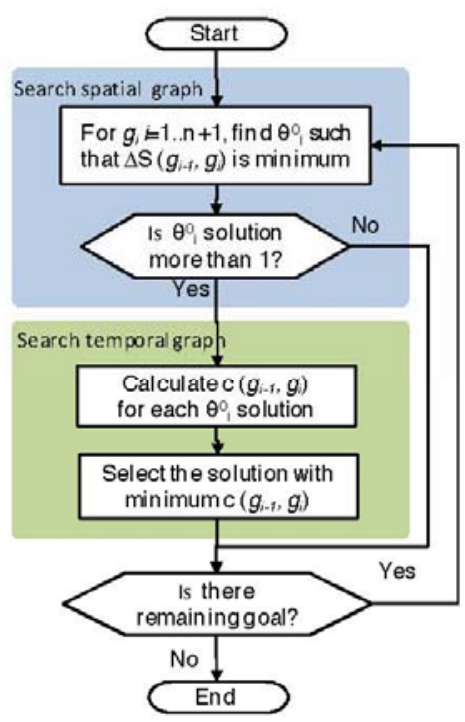

(c) Decision Logic for SMC

Fig. 4 Solution search space representation and decision logic. 
On the other hand, for some $\boldsymbol{t}_{\text {desired }}$ settings, TMC is more practical since it can minimize motion time to satisfy the temporal constraint. These hypotheses are verified in Subsection 5.2. The range of $\boldsymbol{t}_{\text {desired }}$ value that demarks the appropriateness of using one scheme from the other cannot be clearly defined.

Therefore, we performed in that section simulations and evaluated empirical results.

\subsection{STMC: A Cross-Breed Algorithm Utilizing TMC and SMC}

Figure 5 also shows that work cell size and motion time can be conflicting requirements: minimizing motion time influences the resulting work cell size and vice versa. If an exact mathematical function can be well defined to quantify this relationship, it would be a key solution to the problem of minimizing the swept volume and guaranteeing that the $\boldsymbol{t}_{\text {desired }}$ constraint is satisfied. With its absence, an intuitive solution is to utilize the individual advantages of TMC and SMC as hypothesized in Subsection 4.3. Since the $\boldsymbol{t}_{\text {desired }}$ values applicable for TMC and SMC cannot be known without performing optimization, a practical solution is to allocate relevant optimization resource, which is the calculation time in this study. Figure 6 shows a straightforward implementation of this solution. It allocates the calculation time for SMC and TMC using AllotDesignTime() function, and afterwards selects the better solution of the two simulations. STMC denotes an optimization that maxCalcTime is equally divided between TMC and SMC to perform optimization. Further analysis on time resource allocation between SMC and TMC will be considered for future studies. Since the time allocation should not necessarily be halved between SMC and TMC, a heuristic function can be implemented for AllotDesignTime() function. A research can be undertaken to reactively adjust the time allocation based on the monitored performance of SMC and TMC during optimization.

\section{Simulations, results and discussion}

Simulations are conducted to evaluate the proposed optimization framework (Section 5.1), and to determine the applicability of the TMC and SMC under various $t_{\text {desired }}$ constraints (Subsection 5.2) and STMC (Subsection 5.3). The

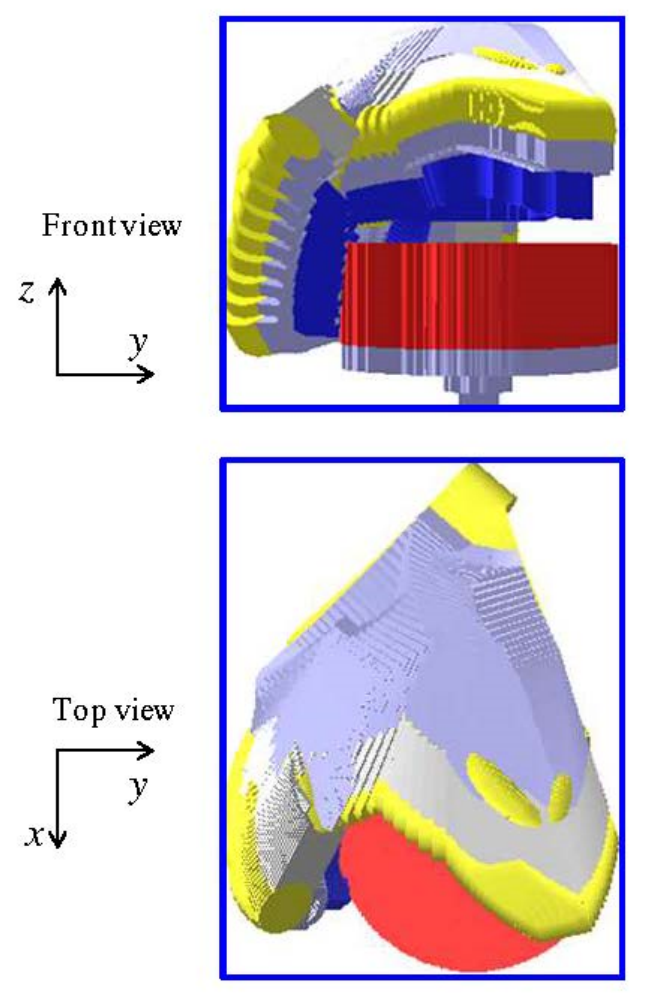

(a) TMC

$$
t_{n+1}=3.022[\mathrm{~s}], f=0.504\left[\mathrm{~m}^{3}\right]
$$
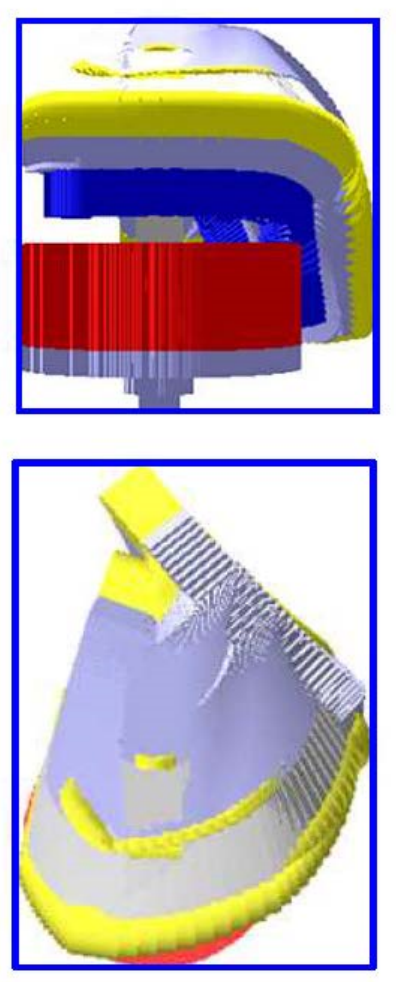

(b) $\mathrm{SMC}$

$t_{n+1}=3.318[\mathrm{~s}], f=0.456\left[\mathrm{~m}^{3}\right]$

Fig. 5 Actual motion and swept volume of the manipulator system employing SMC and TMC. 
table rotation resolution $r$ is 64 , adequate to obtain good quality solutions while $\theta^{0, \min }$ and $\theta^{0, \max }$ are set to $-\pi$ $[\mathrm{rad}]$ and $\pi$ [rad] to cover one rotation. The rotary table location is at $(0,0,0)$ while the initial manipulator base placement is $(-600,0,0)$ based on an empirical method (Gueta et al, 2007). No tool attachment is initially assumed. A calculation time limit of two hours is imposed which is a reasonable length of simulation based on our empirical results. The simulation is conducted using an Intel Core Duo 3.0GHz processor with 4GB memory.

\subsection{Comparison of various optimization frameworks}

Frameworks are compared to evaluate the proposed framework:

- GM: Goal rearrangement and motion coordination

- $\quad$ BGM: Base placement optimization using SA with GM

- TGM: Tool attachment optimization using SA with GM

- $\quad$ BTGM: Base placement and tool attachment optimizations with GM

GM is the reference of comparison since it is the basic framework while BTGM is the proposed framework. To design a compact system, no $\boldsymbol{t}_{\text {desired }}$ is imposed and SMC is employed. Parameter $\mathrm{n}=30$ (CC1), 40 (CC2) and 50 (CC3) as shown in Fig. 7. For each goal setting, 4 instances (i.e., equal number of goals with different positions on B) are simulated. Each instance is run thrice and the best solution is selected.

Figure 8 shows a comparison of reduction in work sell size relative to GM: $23.99 \%$ (BGM), $17.46 \%$ (TGM) and $28.02 \%$ (BTGM), in which higher values are desirable. It shows that the manipulator base placement is very crucial since it defines the floor area occupied by the manipulator system. The performance of TGM shows that a tool attachment can reduce the swept volume by changing the manipulator flange pose. BTGM has the best performance for all goal settings which is expected because of the combined effect of optimizing the base placement and tool attachment.

\subsection{SMC and TMC Evaluation}

Utilizing the proposed framework, SMC and TMC are evaluated to determine their applicability over arrange of $t_{\text {desired }}$ values. In INF setting, no temporal constraint is imposed. For continuity, the same goal settings of the examples in Section 3 and 4 are utilized. This time the base placement and tool attachment optimizations are conducted in the simulations.

Figure 9 shows the result where the blue lines are the relevant data in this section while the red line is discussed in Section 5.3. The result shows that SMC is more appropriate than TMC for $t_{\text {desired }}=3.5$ [s] while TMC only satisfies the constraint $t_{\text {desired }}=3.0[\mathrm{~s}]$. This constraint can be viewed to be too restrictive for SMC. At $t_{\text {desired }}=\mathrm{INF}$, the

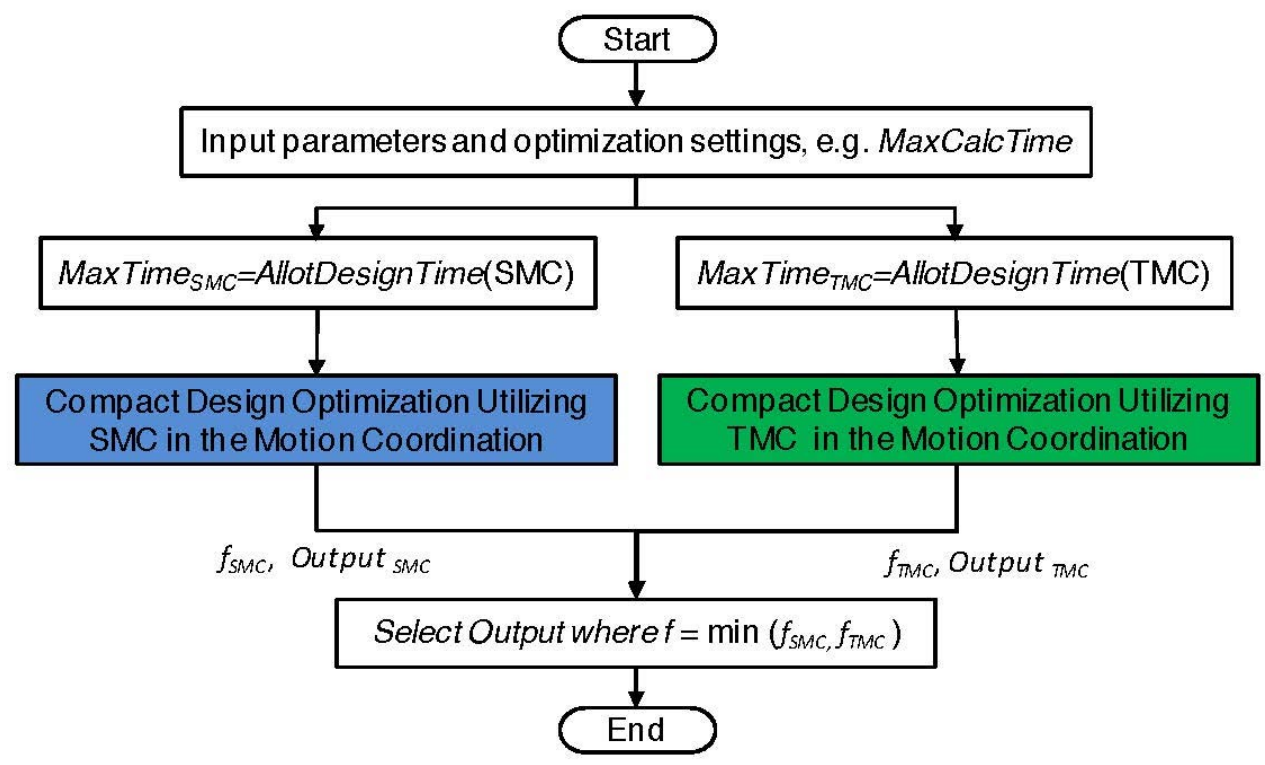

Fig. 6 A cross-breed algorithm utilizing SMC and TMC. 
volume is $0.269\left[\mathrm{~m}^{3}\right]$ and the corresponding motion time is 4.581 [s]. The above result supports our hypothesis that the suitability of TMC and SMC depends on the $t_{\text {desired }}$ value.

The result in Figure 9 is also observed in other goal configurations such that there is always a critical point wherein SMC cannot satisfy a $t_{\text {desired }}$ constraint (Gueta et al., 2009). This point defines the applicable range of TMC and SMC. It is difficult to be determined due to its dependence on goal configuration, manipulator and table parameters.

\subsection{STMC Evaluation}

The proposed STMC is evaluated to determine their applicability over arrange of $t_{\text {desired }}$ values. From the results shown in Fig. 9, STMC is able to satisfy all the temporal constraint settings (from $t_{\text {desired }}=3$ to $t_{\text {desired }}=\mathrm{INF}$ ). In contrast, SMC was not able to satisfy the constraint if $t_{\text {desired }}=3$ [s], which is a limitation of SMC because it only

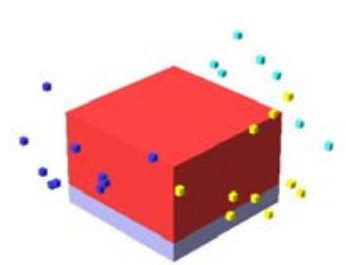

(a) CC1

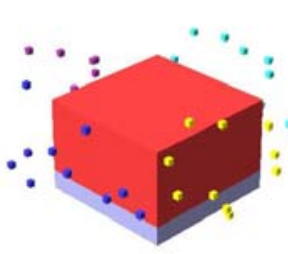

(b) $\mathrm{CC} 2$

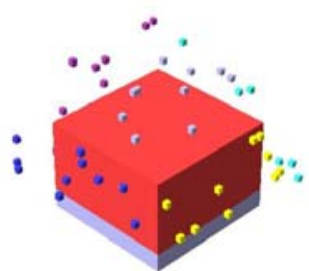

(c) $\mathrm{CC} 3$

Fig. 7 Goal settings.

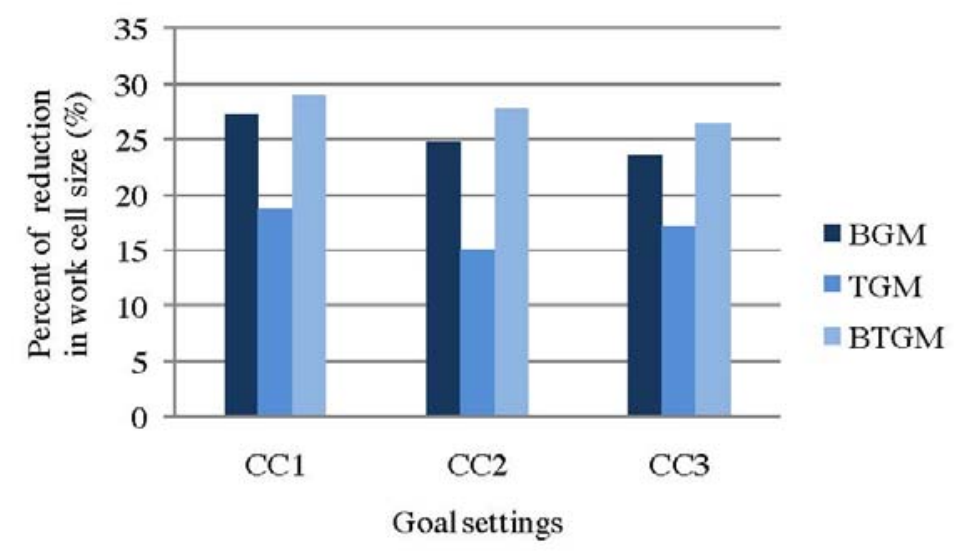

Fig. 8 Performance of optimization frameworks relative to GM.

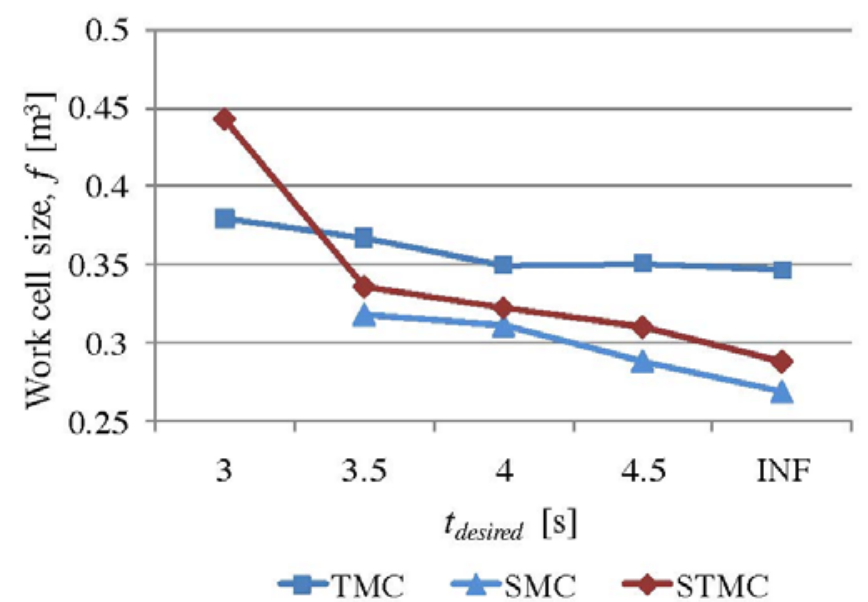

Fig. 9 Swept volume under various $t_{\text {desired }}$ setting 
Gueta, Chiba, Arai, Ueyama, Rubrico and Ota,

Journal of Advanced Mechanical Design, Systems, and Manufacturing, Vol.11, No.2 (2017)

Table 2 Work cell size $f$ for maxCalcTime $\mathbf{2}$.

\begin{tabular}{|c|c|c|c|c|c|}
\hline \multirow{2}{*}{ scheme } & \multicolumn{5}{|c|}{ Desired lead time, $t_{\text {desired }}[\mathrm{s}]$} \\
\cline { 2 - 6 } & 3.0 & 3.5 & 4.0 & 4.5 & INF \\
\hline TMC & $\mathbf{0 . 4 4}$ & 0.43 & 0.38 & 0.38 & 0.35 \\
\hline SMC & $*$ & $\mathbf{0 . 3 4}$ & $\mathbf{0 . 3 2}$ & $\mathbf{0 . 3 1}$ & $\mathbf{0 . 2 9}$ \\
\hline
\end{tabular}

optimizes the work cell size without considering the time constraint. Here care should be taken that the quality of solution of STMC case may be worse compared to using SMC because of the halved running time of SMC in STMC to SMC. In the case of TMC, it satisfies all the time constrains; however for $t_{\text {desired }}=3.5[\mathrm{~s}], t_{\text {desired }}=4[\mathrm{~s}]$ and $t_{\text {desired }}=\mathrm{INF}$, TMC has worse performance that STMC as indicated by larger work cell size. This is an inherent limitation of TMC, because it only optimizes the motion time without considering the resulting work cell size. Based on the above discussion, it can be said that STMC is better than SMC and TMC when both minimal work cell size and temporal constraint have to be considered.

In INF setting, no temporal constraint is imposed. For continuity, the same goal settings of the examples in Section 3 and 4 are utilized. This time the base placement and tool attachment optimizations are conducted in the simulations. The point at which SMC cannot satisfy the constraint in Section 5.2 is difficult to be determined mathematically thereby motivating us to propose STMC. Table 2 shows the optimization result. The highlighted values are selected as the solution, shown as the red line in Fig. 9. In the result, TMC is better than SMC at $t_{\text {desired }}=3.0$ [s]. In other settings, SMC is better than TMC. The difference in the quality of solutions when maxCalcTime $=1[\mathrm{~h}]$ and $2[\mathrm{~h}]$ is not surprising; a longer calculation time can allow more improvement in solution quality. The result shows that dividing the simulation time between SMC and TMC is reasonable to avoid from empirically determining the critical point defining the range of $t_{\text {desired }}$ settings applicable for TMC and SMC.

The applicability of the proposed optimization framework can be extended to other multiple-goal tasks and can be applied to other manipulator systems. For manipulator system with higher dofs (e.g., 6-dof manipulator and 3-dof table), redundancy resolution would be complicated requiring modifications in the motion coordination. Furthermore, the concept of using SMC to minimize swept volume can be extended to other motion planning algorithms.

\section{Summary and conclusion}

This study conducted the following: (1) formulated the compactness measure based on the manipulator swept volume with temporal constraint, (2) introduced a spatial motion coordination (SMC) scheme and evaluated it along with a temporal motion coordination (TMC) scheme to determine their applicability relative to temporal constraint setting (3) proposed a best-effort search methodology to minimize swept volume and satisfy the constraint, and (4) proposed a crossbreed algorithm (STMC) to utilize both schemes and thereby satisfy the constraint.

The integrated base placement optimization, tool attachment optimization, goal rearrangement and spatial motion coordination is the most effective optimization framework resulting into a $28 \%$ reduction in the work cell size compared to performing only goal rearrangement and motion coordination. SMC and TMC performance shows limitation on the temporal constraint setting. STMC is shown to be an effective solution to deal with the limitation.

\section{References}

Cagan, J., Shimada, K., and Yin, S., A survey of computational approaches to three-dimensional layout problems, Computer-Aided Design, Vol. 34, Issue 8 (2002), pp. 597-611.

Grangeon, N., Norre, S., and Tchernev, N., Heuristic-based approach for an industrial three-dimensional layout problem, Production Planning and Control, Vol. 16, Issue 8 (2005), pp. 752-762.

Gueta, L. B., Chiba, R., Ota, J., Arai, T., and Ueyama, T., Design and optimization of a manipulator-based inspection system. the Society of Instrument and Control Engineers Trans. on Industrial Application, Vol. 6, No.7 (2007), pp. 41-51.

Gueta, L. B., Chiba, R., Ota, J., Arai, T., and Ueyama, T., Design of the end-effector tool attachment for robot arm with multiple reconfigurable goals, Proceedings of IEEE International Conference on Automation and Science Engineering, (2008), pp.876-881.

Gueta, L. B., Chiba, R., Ota, J., Arai, T., and Ueyama, T., Compact design of work cell with robot arm and positioning 
table under a task completion time constraint, Proceedings of IEEE International Conference on Intelligent Robots and Systems, (2009), pp.807-813.

Gueta, L. B., Chiba, R., Ota, J., Arai, T., and Ueyama, T., Practical point-to-point multiple-goal task realization in a robot arm with a rotating table, Advance Robotics, Vol. 25, Issue 6-7, (2011), pp. 717-38.

Helsgaun, K., An effective implementation of the Lin-Kernighan traveling salesman heuristic. European Journal of Operations Research, Vol. 126, Issue 1 (2000), pp. 106-130.

Hopp, W. J. and Spearman, M. L., Factory Physics, 3rd Ed., (2008), McGraw Hill.

Hsu, D., Latombe, J.-C., and Sorkin, S., Placing a robot manipulator amid obstacles for optimized execution, Proceedings of IEEE Int. Symposium on Assembly and Task Planning, (1999), pp. 280-285.

Jang, H., Lee, S. and Choi, S., Optimization of floor-level construction material layout using Genetic Algorithms, Automation in Construction, Vol. 16, Issue 4 (2007), pp. 531-545.

Jang, S. - H., A study on three dimensional layout design by the simulated annealing method, Journal of Mechanical Science and Technology, Vol. 22, Issue 11 (2008), pp. 2016-2023.

Jouaneh, M. K., Wang, Z., and Dornfeld, D. A., Trajectory planning for coordinated motion of a robot and a positioning table: part 1- path specification, IEEE Transactions on Robotics and Automation, Vol. 6, No. 6, (1990), pp. 735-45.

Kim, J.-O., and Khosla, P. K., A formulation for task based design of robot manipulators, Proceedings of IEEE/RSJ International Conference on Intelligent Robots and Systems, (1993), pp. 2310-2317.

Kirkpattrick, S., Gelatt, C., and Vechi, M. P., Optimization by simulated annealing, Science, Vol. 220, No. 4598 (1983), pp. 671-80.

Kusiak, A. and Heragu, S. S., The facility layout problem, European Journal of Operations Research, Vol. 29, No. 3 (1987), pp. 229-251.

Lueth, T. C., Automated planning of robot workcell layouts, Proceedings of IEEE International Conference on Robotics and Automation, (1992), pp. 1103-1108.

McKendall Jr., A. R., and Hakobyan, A., Heuristics for the dynamic facility layout problem with unequal-area departments, European Journal of Operational Research, Vol. 201, Issue 1 (2010), pp. 171-182.

Meller, R. D. and Gau, K. -Y., The facility layout problem: Recent and emerging trends and perspectives, Journal of Manufacturing Systems, Vol. 15, Issue 5 (1996), pp. 351-366.

Park, M.- W., and Kim, Y. -D., A systematic procedure for setting parameters in simulated annealing algorithms, Computers Operations Research, Vol. 25, Issue 3 (1998) pp. 207-17.

Robgoderer, U. and Woenckhaus, C., A concept for automatical layout generation, Proceedings of IEEE International Conference on Robotics and Automation, (1995), pp. 800-805.

Saha, M., Roughgarden, T., and Latombe, J.- C., Planning tours of robotic arms among partitioned goals, International Journal of Robotics Research, Vol. 25, No. 3 (2006), pp. 207-224.

Shiller, Z., Optimal robot motion planning and work-cell layout design. Robotica, Vol. 15, Issue 1 (1997), pp. 31-40.

Singh, S. P. and Sharma, R. R. K., A review of different approaches to the facility layout problems. International Journal of Advanced Manufacturing Technology, Vol. 30, Issue 5 (2006), pp. 425-433.

Tay, M. L., and Ngoi, B. K. A., Optimising robot workcell layout, International Journal of Advanced Manufacturing Technology, Vol. 12, Issue 5 (1996), pp. 377-385.

Wang, T. Y., Wu, K. B., and Liu, Y. W., A simulated annealing algorithm for facility layout problems user variable demand in cellular manufacturing systems. Computers in Industry, Vol. 46, Issue 2 (2001), pp. 181-188.

Yagmahan, B. and Yenisey, M. M., Ant colony optimization for multi-objective flow shop scheduling problem, Computers and Industrial engineering, Vol. 54, Issue 3 (2008), pp. 411-420.

Yeung, L. H. W., and Tang, W. K. S., A hybrid genetic approach for container loading in logistic industry, IEEE Transaction on Industrial Electronics, Vol. 52, Issue 2 (2005), pp. 617-627. 